\title{
Selbstevaluation als relevante Komponente der Identität und des Selbst(Konzepts) der Lernenden im Fremdsprachenunterricht
}

\section{Jana Veličková}

The present text explores the questions of how identity, an always present property in foreign language learners, is expressed, how the concepts of identity and self interact and what kind of interplay between the concept of identity and self (concept) takes place in foreign language learning and teaching, and which role the self-evaluation of (foreign language) learners plays in this context. The aim of the text is to review the level of knowledge about these concepts and, moreover, to specify the theoretical basis for further empirical investigation in this area.

Self-Evaluation, Identity, Self(Concept), Foreign Language Learning and Teaching, Multilingualism

Der vorliegende Text untersucht, wie Identität, ein stets präsentes Attribut von Fremdsprachenlernern, beim Fremdsprachenlernen zum Ausdruck kommt, und erläutert darüber hinaus die Fragen, wie das Wechselspiel zwischen Identität und Selbst(Konzept) im Fremdsprachenunterricht aussieht und welche Bedeutung in diesem Kontext der Selbstevaluation der (Fremdsprachen)Lernenden zukommt. Das Ziel des Textes besteht darin, den Erkenntnisstand zu diesen Konzepten aufzuarbeiten und darüber hinaus die theoretischen Grundlagen für weitere empirische Untersuchungen in diesem Gebiet zu präzisieren.

Selbstevaluation, Identität, Selbs(Konzept), Fremdsprachenunterricht, Mehrsprachigkeit

\section{Einführung}

Im Einklang mit den Tendenzen der Fremdsprachenlehr- und -lernforschung in den letzten Jahrzehnten kommt der vorliegende Text dem Bedarf nach gründlicher Fokussierung auf individuell spezifische Variablen entgegen und thematisiert die fächerübergreifenden, auf die einzelnen Individuen bezogenen Konstrukte „Identität“, „Selbst“ und „Selbstevaluation“. Es handelt sich zwar um ursprünglich aus der Psychologie stammende Konzepte, diese haben sich jedoch inzwischen auch in der Empirie und Praxis der Fremdsprachendidaktik fest etabliert.

Das Ziel dieses Textes besteht in der Aufarbeitung des Erkenntnisstandes zu diesen Konzepten bzw. zu den Schnittstellen zwischen ihnen und im Aufzeigen von möglichen Diskrepanzen oder auch Übereinstimmungen in diesem Geflecht. In erster Linie soll der Text dazu beitragen, die theoretischen Grundlagen für die weitere empirische Untersuchung der Selbstevaluation als einer Komponente der Identität und des Selbst(Konzepts) der Lernenden ${ }^{1}$ im Kontext des Fremdsprachenlernens

1 In diesem Text wird aus Gründen der besseren Lesbarkeit darauf verzichtet, geschlechtsneutrale Formulierungen zu verwenden. Die personenbezogenen Bezeichnungen in männlicher Form beziehen sich demnach sowohl auf Männer als auch auf Frauen. 
zu präzisieren. Der Fokus liegt demnach vornehmlich auf der Begriffsbildung und der möglichen Operationalisierung der angeführten Konzepte für die Forschung im Rahmen der Fremdsprachendidaktik. ${ }^{1}$

Um dieses Ziel zu erreichen, werden zuerst die behandelten Begriffe (als mögliche Objekte der Begriffsbildung im Rahmen der Fremdsprachenlernforschung) voneinander abgegrenzt. Anschließend werden Konzepte wie Identität, Selbst(Konzept) und Selbstevaluation vor dem spezifischen Hintergrund des Fremdsprachenunterrichts diskutiert.

\section{Abgrenzung des Begriffs Selbstevaluation}

Die Selbstevaluation (self-assessment, self-evaluation) stellt im Kontext des vorliegenden Textes eine fächerübergreifende Variable dar, die vor dem Hintergrund anderer Konzepte reflektiert wird. Obwohl der Text pädagogisch ausgerichtet ist, wird es unumgänglich sein, sich nicht nur bei der terminologischen Abgrenzung dieses zentralen Begriffes immer wieder psychologischer Annahmen zu bedienen, weil die Selbstevaluation primär im Bereich der Psychologie anzusiedeln ist.

Problematisch dabei ist, dass mit dem Begriff "Selbstevaluation“ - je nach dem fachlichen Kontext, in dem er verwendet wird - verschiedene Vorstellungen assoziiert sind. In der Psychologie wir unter „Selbstevaluation“ im Allgemeinen das Bild verstanden, das sich ein Individuum von sich selbst macht (z. B. das Bild eines hervorragenden Schülers). In diesen Zusammenhang fallen Begriffe wie „das Selbst“, „das Selbstbild“, „die Vorstellung von den eigenen Fähigkeiten“ oder „die Selbstwirksamkeit“ (vgl. Moschner und Dickhäuser 2010). Genaueres dazu findet sich in Kap. 4 der vorliegenden Überblicksdarstellung. In der Pädagogik wird unter „Selbstevaluation" dagegen vornehmlich eine bestimmte metakognitive Lernstrategie verstanden, die ein integraler Bestandteil des selbstgesteuerten (self-directive learning) und selbstregulierten (self-regulated learning) Lernens ist (Janíková 2007; Panadero und Alonso-Tapia 2013). So halten etwa Brunstein und Spörer (2010: 752) die Selbstevaluation für eine wichtige Bedingung für lernerzentriertes Lernen.

Innerhalb der Pädagogik wird die Selbstevaluation auch als eine Fähigkeit (Weskamp 2007: 382) bzw. eine Kompetenz beschrieben (Wilkening 2013: 13). Die Fähigkeit zur Selbstevaluation ist nach Dollase (2012: 14) nicht angeboren, sondern wird erst durch die Interaktion mit anderen im Laufe des Lebens erworben. Die Entfaltung der Fähigkeit bzw. Kompetenz zur Selbstevaluation wird also wesentlich durch das soziale Umfeld mitgeprägt. Neben der Bezeichnung „Fähigkeit“ wird auch die Bezeichnung "Fertigkeit" verwendet, wodurch die Wichtigkeit einer angemessenen Entfaltung der Fähigkeit zur Selbstevaluation betont wird (Slavík

1 Es wird also kein Anspruch auf Vollständigkeit erhoben, sondern bewusst der Fokus auf die Schnittstellen dieser Konzepte gelegt. 
1999: 22). Im Hintergrund steht dabei die Vorstellung, dass „Fähigkeiten“ etwas Gegebenes sind, während „Fertigkeiten“ bewusst entwickelt werden müssen.

Der adäquate Bildungsprozess ist durch zwei Faktoren bedingt: einen kognitiven Faktor, der vom Individuum abhängt (vgl. Koštálová, Miková und Stang 2008: 63-65), und den Faktor der sozialen Interaktion, wobei einige Autoren (z. B. Stranovská 2012) die Ansicht vertreten, dass auch die kognitive Entwicklung wesentlich von den sozialen Bedingungen mitgeprägt wird.

Die Selbstevaluation (als selbstregulatorischer Lernprozess) wird jedoch andererseits auch durch die Selbstevaluation als Bild, das der Lerner von sich selbst hat, beeinflusst (vgl. Janíková 2013). Dadurch wird die Selbstevaluation im Sinne der Psychologie zu einem Faktor, der auf den Lernprozess insgesamt Einfluss nimmt.

Im Fremdsprachenunterricht und in der Fremdsprachendidaktik taucht das Konzept der Selbstevaluation seit dem Ende der 1970er Jahre auf (Wolff 1994 zit. nach Weskamp 2007: 382). Im Zentrum der Aufmerksamkeit von Forschung und Praxis steht dieses Konzept dann v. a. seit den 1980er Jahren, vorwiegend im Zusammenhang mit der Entwicklung des Europäischen Sprachenportfolios (Weskamp 2001: 179), das die Selbstevaluation als ein wichtiges Instrument zur Förderung der Lernerautonomie betrachtet. Die Selbstevaluation wird im Fremdsprachenunterricht und in der Fremdsprachendidaktik dadurch zum grundlegenden Baustein der Lernerautonomie (Tassinari 2010). Gleichzeitig wird die Fähigkeit zur Selbstevaluation auch als eine wichtige Voraussetzung für das lebenslange Lernen angesehen (Vollmer 2007: 369), denn sie trägt dazu bei, dass die Lernenden größere Verantwortung für ihr eigenes Lernen übernehmen (Little und Perclová 2001).

Zusammengefasst handelt es sich bei „Selbstevaluation“ um ein relativ komplexes Phänomen. Das Ziel des vorliegenden Beitrags ist dementsprechend nicht, alle möglichen Facetten des Konzepts auszuleuchten, sondern nur diejenigen Aspekte zu erfassen, die für das Verständnis der Stellung von Selbstevaluation innerhalb des Selbstkonzepts bzw. der Identität der Fremdsprachenlerner innerhalb der psychologischen Herangehensweise wichtig sind.

\section{Zum Begriff Identität}

Nicht nur bei Selbstevaluation handelt es sich um eine sozial konstruierte Erscheinung, sondern auch Identität wird erst durch soziale Interaktionen entwickelt (Legutke 2013: 153). Während die Identität einer Person früher für relativ unveränderlich gehalten wurde (vgl. Kresic 2006: 10), überwiegt heute die Auffassung, dass es sich „nicht [um eine] stabile, sondern [um eine] dynamische, sich je nach situativer Umgebung und/oder im zeitlichen Verlauf verändernde und anpassende, sich in Interaktionen und innerhalb der lebensweltlichen Umgebung konstruierende und konstituierende Entität" (Riemer 2013: 223) handelt. 
Der Prozess der Herausbildung der eigenen Identität verläuft durch mehr oder weniger bewusste „[...] Wahrnehmung von Identität bzw. Eindrucksbildung in Bezug auf andere Personen und Gruppen einerseits ${ }^{1}$ und der planvollen Selbstdarstellung gegenüber der ungeplant-unbewussten Herausbildung von Identität andererseits" (Kresic 2006: 28). Eine wichtige Rolle in diesem Prozess spielt die Sprache, denn die Konstruktion der eigenen Identität ist eng an die Sprache als ein Medium der Kommunikation gebunden (zur Rolle der Sprache innerhalb der Identitätsentwicklung im Kontext des Fremdsprachenlernen, s. Roche 2013). In der Psychologie und Soziologie wird Identität als ein ,interaktiv-dynamisch und kommunikativ ausgehandeltes Konstrukt" verstanden (Kresic 2006: 41), dessen Konstruktcharakter am ehesten von konstruktivistischen Identitätskonzepten erfasst wird (mehr dazu in Kresic 2006). Diese Auffassung setzt sich in letzter Zeit immer stärker durch, v. a. im Zusammenhang mit aktuellen soziokulturellen Ansätzen zum Fremdsprachenlernen, die auf Kosten von psycholinguistischen und kognitionswissenschaftlichen Modellen heute immer mehr an Boden gewinnen (vgl. Riemer 2013: 224).

Wie man den obigen Ausführungen entnehmen kann, handelt es sich bei „Identität“ um ein kompliziertes interdisziplinäres Konzept, das sich in seiner Vollständigkeit nur schwierig erfassen und abgrenzen lässt. Da die Identität in diesem Beitrag lediglich den Rahmen darstellt, in dem die Selbstevaluation als ein sich (nicht nur) beim Fremdsprachenlernen abspielender Prozess verankert ist, wird hier auf eine genaue Darstellung der Vielschichtigkeit der einzelnen Auffassungen verzichtet und der Fokus hauptsächlich auf das Verhältnis von Identität, Selbst und Selbstevaluation gerichtet. Eine der Schnittstellen zwischen diesen Konzepten ist die mehr oder weniger bewusst ablaufende Selbstreflexion. Diese wird als eine Fähigkeit verstanden, „Vorstellungen über die eigene Person [zu] entwickeln“ (Riemer 2013: 223). Aus der Fachliteratur geht hervor, dass die Identität einer Person und die Veränderungen, denen sie ggf. unterliegt, dank der Fähigkeit zur (Selbst)Reflexion ins Bewusstsein gehoben werden können. Der Theorie zur Entwicklung der Selbstreflexion bzw. Selbstevaluation ${ }^{2}$ kann man des Weiteren entnehmen, dass die Herausbildung beider unter dem ständigen Einfluss von externen Rückmeldungen stehen, die zu ihrer angemessenen Entfaltung beitragen und daher auch die Herausbildung der Identität mitbestimmen (vgl. z. B. Panadero und Alonso-Tapia 2013). Durch den Kontext des (Fremdsprachen)Unterrichts bedingt, hebt dieser Umstand die Bedeutung eines adäquaten Lehrerfeedbacks hervor, das wiederum als wichtiger Faktor für die Entfaltung der Identität der Lerner gilt.

$1 \mathrm{Im}$ Zusammenhang mit dem sozialen Aspekt der Identitätsbildung wird von der kulturellen Dimension der Identität gesprochen (vgl. Friedrich 2008). Dazu mehr in Kapitel 5.

2 Die (Selbst)Reflexion wird als eine komplexere und umfassendere geistige Aktivität als die (Selbst) Evaluation verstanden. 
Man kann also bereits hier bei der Identitätsbildung und bei der Konstituierung der Selbstevaluation Ähnlichkeiten feststellen, denn beide Konzepte werden wesentlich durch das Interagieren mit dem sozialen Umfeld mitgeprägt, wobei die Selbstreflexion ein breites Spektrum der Identitätskomponenten eines Fremdsprachenlernenden beeinflussen kann (mehr zu diesen Komponenten dazu in Kapitel 5).

Bei der empirischen Untersuchung der Selbstevaluation als einer Komponente der Identität der Lernenden muss demnach auch der Einfluss des sozialen Umfelds beachtet, als eine wichtige intervenierende Variable ausführlich beschrieben und bei der Analyse berücksichtigt werden. Nicht zuletzt darf auch die Lehrerperson mit ihren Rückmeldungen auf die Identitätsbildung und somit die Entwicklung der Selbstevaluation der Lernenden nicht außer Acht gelassen werden.

\section{Selbst(Konzept)}

Wie den obigen Ausführungen $\mathrm{zu}$ entnehmen ist, gibt es einen engen Zusammenhang zwischen der Identität und dem Selbst(Konzept) der Lernenden (self-concept, L2 self), der jedoch nicht ganz einfach zu erfassen und zu definieren ist, da es je nach dem Prisma der unterschiedlichen Paradigmen verschiedene Auffassungen gibt, die jedoch auch gewisse Gemeinsamkeiten aufweisen. In Anlehnung an Haußer (1995) werden nach Kresic (2006: 65) in der Psychologie für das Konstrukt „Identität“ die Termini „Selbst“ bzw. „Selbstkonzept“ gegenüber dem Begriff „Identität“ bevorzugt. Die Termini „Identität“ und „Selbst“ werden jedoch meist synonym gebraucht. Genauso gibt es keinen einheitlichen Usus bei der Verwendung der Termini „Selbst“ und „Selbstkonzept“. Im vorliegenden Text neige ich der Ansicht von Legutke (2013: 156) zu, der der Identität eine übergeordnete Stellung beimisst. Diese lässt sich für ihn als Ergebnis eines dynamischen Verhältnisses aller Erscheinungsformen des Selbst bezeichnen, wobei das Selbstkonzept hier als eine der Dimensionen des Selbst erscheint. Die Forschergruppe um Neisser (1988; Neisser und Jopling 1997, zit. nach Legutke 2013: 154-156) hat in diesem Kontext einen wichtigen Beitrag geleistet, indem sie nachgewiesen hat, dass ,[...] die Herausbildung von Selbstbewusstsein, Wissen über das Selbst, davon abhängt, wie wir andere in unserer Umgebung wahrnehmen, konstruieren und deuten" (Neisser und Jopling 1997, zit. nach Legutke 2013: 154). In Anlehnung daran erarbeitet die Gruppe fünf Erscheinungsformen des Selbst (five selves), für die sie auch jeweils die Bedeutung für das Fremdsprachenlernen bestimmt. Diese fünf Erscheinungsformen bezeichnen die Autoren als

(1) das ökologische Selbst (ecological self),

(2) das interpersonale Selbst (interpersonal self),

(3) das erweiterte Selbst (extended self), 
(4) das private Selbst (private self) und

(5) das Selbstkonzept bzw. das konzeptuelle Selbst (conceptual selfl reflexive self).

Die erste Erscheinungsform, das ökologische Selbst, entwickelt sich aufgrund der physischen und psychischen Interaktionen des Individuums (des Sprachnutzers) mit der Umgebung, wobei diese Interaktionen mittels der Sprache realisiert werden. Es geht also einerseits um die körperliche Fähigkeit, überhaupt in der Fremdsprache zu kommunizieren, andererseits spielen auch die mit der Sprachverwendung verbundenen Gefühle eine Rolle. Sowohl das ökologische als auch das sog. interpersonale Selbst, der zweite Aspekt des Selbst, formieren sich bereits in der Kindheit.

Die Entwicklung des interpersonalen Selbst beruht auf dem „[...] Austausch sprachlicher und parasprachlicher Signale in der Kommunikation" (Legutke 2013: 155). Bei den erwähnten parasprachlichen Signalen handelt es sich also um intersubjektive Sprachhandlungen. Von dem mehr oder weniger regelgerechten Einsatz solcher Signale hängt es ab, welche Erfahrungen der Sprecher beim Kommunizieren macht.

Aus den Erfahrungen (nicht nur im Zusammenhang mit der Kommunikation, sondern auch mit anderen Tätigkeiten und Erlebnissen) kristallisiert sich das erweiterte Selbst heraus, durch das das Individuum auch im weiteren Fortgang des Lebens geprägt wird. Wenn man diesen Aspekt des Selbst konkret auf das Fremdsprachenlernen bezieht, wirkt es sich auf die Art und Weise aus, wie man verschiedene individuelle Tätigkeiten und Arbeitsweisen beim Sprachenlernen ausübt. $\mathrm{Ob}$ man z. B. einen Inhalt mündlich oder schriftlich wiedergeben will, hängt von den individuellen Schemata ab, die in uns verankert sind und die durch unsere Erlebnisse geformt werden.

Die vierte Form des Selbst wird das private Selbst genannt. Genau wie die anderen Ausprägungen geht auch dieses Selbst auf individuelle Erfahrungen zurück, entwickelt sich jedoch stark im Prozess der Bewusstwerdung der „Differenz und Einmaligkeit“ unserer eigenen Erfahrungen. Im Kontext des Fremdsprachenunterrichts tritt dieses Selbst vor allem bei Rollenspielen in Erscheinung, wenn man sich hinter einer Maske verstecken kann.

Als die fünfte und letzte Erscheinungsform des Selbst arbeitet die Forschungsgruppe um Neisser das sog. Selbstkonzept bzw. das konzeptuelle Selbst heraus. Dieses nimmt auch im Rahmen des Identitätskonzepts eine Sonderstellung ein, denn es ist den anderen Bestandteilen des Selbst übergeordnet und überwacht dessen andere Formen. Im Rahmen des konzeptuellen Selbst, so die Autoren der Gruppe, „[....] befasst sich das Individuum mit seinen Erwartungen, seinen Zielen, seinem Engagement. Es reflektiert seine Rollen im sozialen Miteinander, wägt Einflussmöglichkeiten ab, bewertet Fortschritte und Anforderungen " (Legutke 2013: 155). Diese Aussage weist darauf hin, dass das konzeptuelle Selbst der Metaebene angehört. Auf den Unterricht bezogen kommt dieses Selbst bei allen 
reflexiven Handlungen (seien es auch unbewusste) zum Ausdruck, im engeren Sinne aber z. B. bei verschiedenen Aktivitäten zur Selbstevaluation von Lernenden, die sich etwa auf den Lernerfolg oder den Sprachstand, auf die Zielsetzung, die Planung der Lernwege bzw. Strategien, wie man das Ziel erreichen kann, auf den Lernprozess oder direkt auf die Auswertung der gesetzten Ziele und der zu deren Erreichung eingeschlagenen Wege beziehen können.

Zusammenfassend kann die Identität also als ein übergeordnetes Konzept bezeichnet werden, das u. a. aus dem Selbstkonzept (d. h. der Vorstellung, die sich das Individuum von sich selbst macht) besteht. Dieses Selbstkonzept tritt in fünf Erscheinungsformen auf, wobei die Fähigkeit der Selbstevaluation bzw. Selbstreflexion mit dem sog. konzeptuellen Selbst zusammenhängt. Das Wissen des Forschers von dieser Verankerung der Selbstevaluation im konzeptuellen Selbst kann nicht nur zu einem besseren Verständnis der psychologischen Vorgänge beim Lernen, sondern auch zu einem präziseren Forschungsdesign bei deren Erforschung beitragen. Da es offenbar schwierig ist, die jeweiligen Erscheinungsformen des Selbst voneinander zu trennen, sollte bei der empirischen Untersuchung des konzeptuellen Selbst, das ja auch die Fähigkeit zur Selbstevaluation umfasst, gleichzeitig auch auf die Charakteristika der anderen Erscheinungsformen des Selbst (z. B. auf den Lernstil und die Erfahrungen aus den vorangegangenen Sprachhandlungen) eingegangen werden.

\section{Mehrsprachigkeit beim Fremdsprachenlernen}

Geht man von den Ausführungen der vorigen Kapitel aus, kann man sicher nicht daran zweifeln, dass der Fremdsprachenunterricht dank der zahlreichen Möglichkeiten der Interaktion zwischen dem Individuum und seinem sozialen Umfeld ein einzigartiges Potenzial zur Identitätsentfaltung, Entwicklung des Selbstkonzepts und damit auch zur Entwicklung der Fähigkeit zur Selbstevaluation bietet, die alle zu empirischen Untersuchungen einladen.

Darüber hinaus hat man es beim Fremdsprachenlernen allerdings außerdem noch mit einem ganz spezifischen Kontext zu tun, und zwar mit dem der Mehrsprachigkeit, in dem nebeneinander zwei und mehr Sprachen koexistieren. Dieser spezifische Kontext wirkt auch auf die Identität der Lernenden, denn die Sprache an sich ist ein wichtiges Element der Identitätsbildung. Einerseits wird die Identität der Lernenden durch kognitive Faktoren beeinflusst, andererseits spielen das Prestige bzw. der Ruf und der Marktwert der zu lernenden Sprache eine wichtige Rolle (vgl. Oppenrieder und Thurmair 2003: 53).

Die mit der Kognition verbundenen Faktoren betreffen auf der einen Seite den Stand der kognitiven Entwicklung des Lerners, auf der anderen das vorhandene Sprachvermögen, d. h. das Sprachwissen (Grammatikregeln, Wortschatz und soziolinguistische Konventionen) sowie das Sprachkönnen (die Fähigkeit, in der 
Fremdsprache zu kommunizieren). Da man auf niedrigeren Sprachniveaus die Sprache noch nicht in ausreichendem Maße beherrscht, um sich im Einklang mit dem Stand der eigenen kognitiven Entwicklung adäquat auszudrücken, wie dies in der Muttersprache ganz selbstverständlich der Fall ist, kann es zu negativen Auswirkungen auf die Lerneridentität kommen. Der Lernende muss sich in seinen Äußerungen auf das vorhandene Sprachenvermögen beschränken und demnach in der Kommunikation manchmal eine untergeordnete Rolle (wie die eines Kindes) einnehmen, die nicht seiner tatsächlichen kognitiven Entwicklung entspricht (Kleppin 2013: 92). Der Lernende, der noch über kein so breites Sprachrepertoire verfügt, muss häufig auf Äußerungen verzichten, die einen hohen Komplexitätsgrad aufweisen. Kleppin (2013) führt in diesem Zusammenhang den Begriff Fremdsprachenlerneridentität(en) ein, den sie mit der veränderten Rolle in Zusammenhang bringt, die Menschen aus den genannten Gründen in einer Fremdsprachenlernsituation einnehmen.

Ein anderer identitätsstiftender Aspekt ist das subjektiv wahrgenommene Prestige der Sprache. Dieses scheint auf die idealisierte Vorstellung von sich selbst einen bedeutenden Einfluss auszuüben, denn es bezieht sich auf eine Gruppe (bzw. Gruppen), der (denen) man gerne angehören möchte (Riemer 2013: 223). Im diskutierten Kontext kann man darunter die Sprachgemeinschaften (auf der Makroebene z. B. die Deutschen, auf der Mikroebene z. B. eine konkrete Gruppe von Austauschpartnern aus einem deutschsprachigen Land) verstehen. Dörnyei (2005: 105-106) fügt der Vollständigkeit halber noch das idealisierte L2-Selbstkonzept (ideal L2 self) hinzu, das den motivierten Lernenden dabei hilft, das aktuelle Selbst mit dem idealisierten in Übereinstimmung zu bringen.

Diese eher intrinsisch orientierten motivationalen Beweggründe zum (Fremdsprachen-)Lernen haben ihr extrinsisches Pendant: das Soll-Selbstkonzept (ought-to L2 self), das durch fremdbestimmte Regulation geprägt ist. Das Fremdsprachenlernen ist also ein Spielfeld, auf dem auf der einen Seite das aktuelle und auf der anderen das angestrebte (idealisierte und Soll-) Selbstkonzept der Lernenden steht. Die Lernenden stehen unter dem Einfluss von endogenen (Lerner mit seiner Identität) und exogenen (Lernumgebung) Faktoren. Damit sie die Fremdsprache erlernen, müssen sie diese Diskrepanz durch ihre eigene Willenskraft und Motivation ausbalancieren. Im Zusammenhang damit lässt sich darauf hinweisen, dass die L2-Identitätsforschung häufig mit der L2-Motivationsforschung eng kooperiert, denn für die Motivationsforschung stellt das Selbstkonzept der Lernenden die zentrale Kategorie dar, weil, wie Riemer (2013: 227) behauptet, Identität einen bedeutenden Einfluss auf die Motivation der Lernenden zum Fremdsprachenlernen hat.

Man befindet sich also als Fremdsprachenlerner ständig in einem Rollenspiel, in welchem einerseits eigene Erwartungen vorhanden sind, andererseits aber auch das soziale Umfeld, seien es die Lehrkräfte oder die Sprecher der Zielkultur, die ebenfalls 
ihre Erwartungen geltend machen, seine Erwartungen geltend macht. Im Rahmen des sozialen Umfelds spielt die Lehrkraft mit ihrem Feedbackverhalten eine besonders wichtige Rolle, weil sie eben durch dieses Feedback wesentlich zum Erfolgsgefühl und zur Motivation der Lernenden beitragen kann (Riemer 2013: 228).

Für die Erforschung der Selbstevaluation vor dem Hintergrund der Identität der Fremdsprachenlernenden ergibt sich aus der Diskussion in diesem Abschnitt, dass bei der Analyse der in der Fremdsprache formulierten Selbstevaluationsäußerungen auch die soziale Bewertung der Sprache und das jeweilige Sprachniveau berücksichtigt werden sollten, die beide die Artikulation der Selbstevaluation wesentlich prägen können. Gleichzeitig sollte dabei die Erfassung der Motivation der Lernenden nicht übergangen werden.

\section{Rolle der Lernertexte}

Mit Blick auf die Empirie soll abschließend noch auf das Potenzial von Lernertexten bei der Erforschung der Identitätsbildung und des Selbstkonzeptes der Lernenden eingegangen werden, denn nach Legutke (2013: 158) gelten Lernertexte (sowohl mündliche als auch schriftlich fixierte) als „Manifestationen der Dimensionen des Selbst", das durch den diskursiven, textuellen oder didaktischen Zugriff entschlüsselt werden kann (Legutke 2013: 158-159). Als eine spezielle Art von Lernertexten kann man auch die Selbstevaluationstexte der Lernenden bezeichnen. Bei der Erforschung der Selbstevaluation der Lernenden stellt die Analyse der Manifestationen des Selbst bzw. der sprachlichen Konstituierung der Identität anhand identitätsmarkierender Formulierungen in Selbstevaluationstexten ein interessantes Forschungsfeld dar.

Die Selbstevaluation stellt nicht nur wertvolles Material (die Selbstevaluationstexte der Lernenden) für empirische Untersuchungen zur Verfügung, sondern ist auch eine der wichtigen Methoden zur empirischen Untersuchung der Identitätsentwicklung bei Fremdsprachenlernenden (Legutke 2013: 154).

\section{Fazit}

Der vorliegende Text verfolgte einige der Forschungstendenzen der letzten Jahre auf dem Gebiet des selbstgesteuerten bzw. selbstorganisierten Lernens, die als Forschungsgegenstand das „Selbst“ fokussieren. Gegenstände des Textes waren die Konzepte Identität und Selbst(Konzept) in Bezug auf das Fremdsprachenlernen, vor deren Hintergrund das Konstrukt der Selbstevaluation reflektiert wurde. Ziel dieses Textes war es, den Erkenntnisstand zu diesen Konzepten bzw. zu den Schnittstellen zwischen ihnen aufzuarbeiten, die Diskrepanzen und Stimmigkeiten in diesem Geflecht aufzuzeigen und zur Begriffsbildung der genannten Konzepte im Rahmen der Fremdsprachenlernforschung beizutragen. 
Die Abgrenzung der angeführten Konzepte voneinander erwies sich teilweise als unscharf, denn es besteht eine enge Verbindung zwischen allen behandelten Konstruktenundeshängtvomjeweiligen Forschungsvorhabenab, welcheTiefenschärfe angestrebt wird. Die präsentierten Begriffsbestimmungen sollten demnach nicht als musterhaft, sondern als ein möglicher Ausgangspunkt unter anderen für die Begriffsbildung bei Untersuchungen im Rahmen der Fremdsprachenlernforschung betrachtet werden. Neben den theoretischen Ausführungen zu den Variablen wurde daher auf Implikationen für die Forschung hingewiesen, die aus der Verankerung der Selbstevaluation im Konstrukt der Identität und von dessen unabdingbarem Bestandteil - dem Selbst(Konzept) hervorgehen. So ist es beim Konzipieren vom Forschungsvorhaben im Bereich des Fremdsprachenlernens unumgänglich, die jeweiligen Konzepte getrennt vom Kontext zu untersuchen. Konkret betreffen die zu berücksichtigten Implikationen exogene (im Umfeld des Lernens) und endogene (im Lerner verankerte) Faktoren. Unter den exogenen Faktoren werden insbesondere das soziale Umfeld der Lerner und die Sprache, die gelernt wird, verstanden. Zum sozialen Umfeld gehören im Kontext des Fremdsprachenunterrichts vor allem die Lernergruppe und die Lehrperson, welche die Identitätsentwicklung des Individuums durch die Interaktion mit ihm und ihr Feedbackverhalten in nicht geringem Maße prägen. Im weiteren Sinne gehören zum sozialen Umfeld aber auch gesamtgesellschaftliche Kräfte, die den (mehr oder weniger subjektiv empfundenen) Marktwert und die Reputation der zu lernenden Sprache (und der jeweiligen L1 der Lerner) bestimmen. Den exogenen Faktoren stehen die endogenen Faktoren gegenüber, zu denen nicht nur die Identität des Lerners, sondern auch seine kognitiven Fähigkeiten und sein Sprachenvermögen gehören.

\section{Literaturverzeichnis}

Brunstein, Joachim C. / Spörer, Nadine (2010): Selbstgesteuertes Lernen. In: Rost, Detlef H. (Hg.): Handwörterbuch Pädagogische Psychologie. Weinheim und Basel, Beltz Verlag. S. 753-754.

Casas, Alfonso Mejía (2013): Wenn und wie Lernerrollen ausgehandelt werden. Eine Studie zu den Bedingungen, Handlungsstrategien und Konsequenzen der Förderung der Lernerautonomie im Fremdsprachenunterricht in Kolumbien. Diss. Bielefeld, Universität Bielefeld.

Dollase, Rainer (2012): Kritik der Qualitätssicherung. Bürokratische, sinnlose und sinnvolle Wege zu mehr Qualität. In: Profil 7-8/2012. S. 12-27.

Dörnyei, Zoltán (2005): The psychology of the language learner: individual differences in second language acquisition. New York, Lawrence Erlbaum Associates.

Friedrich, Gila (2008): Identität - ein geschichtsloses Konstrukt? Pädagogische Überlegungen zum Identitätsbegriff einer technisierten und zunehmend digitalisierten Kultur. Münster, LIT Verlag.

Hallet, Wolfgang (2013): How to do identity: Autobiographische Akte im Fremdsprachenutenunterricht. In: Burwitz-Melzer, Eva / Königs, Frank G. / Riemer, Claudia (Hgg.): Identität und Fremdsprachenlernen. Tübingen, Gunter Narr. S. 58-68. Haußer, Karl (1995): Identitätspsychologie. Berlin, Springer. 
Hufeisen, Britta (2013): „Ich lerne (Fremd-)Sprachen also bin ich?“ Sprachenlernen und Identität im Fremdsprachenunterricht. In: Burwitz-Melzer, Eva / Königs, Frank G. I Riemer, Claudia (Hgg.): Identität und Fremdsprachenlernen. Tübingen, Gunter Narr. S. $80-89$.

Janíková, Věra (2007): Autonomni učeni a lexikálni strategie při osvojováni cizich jazyků. Brno, Masarykova univerzita.

Janíková, Věra. (2013): Mnohojazyčnost a didaktika cizich jazykü. Brno, Masarykova univerzita. Kleppin, Karin (2013): Werde ich jemand anders, wenn ich eine neue Sprache lerne? Fremdsprachenlerneridentität(en), ein sinnvoller Begriff? In: Burwitz-Melzer, Eva / Königs, Frank G. / Riemer, Claudia (Hgg.): Identität und Fremdsprachenlernen. Tübingen, Gunter Narr. S. 90-99.

Knoll, Jörg (2001): Wer ist das „Selbst“? In: Dietrich, Stephan (Hg.): Selbstgesteuertes Lernen in der Weiterbildungspraxis. Ergebnisse und Erfahrungen aus dem Projekt SeGeL. Bielefeld, W. Bertelsmann. S. 201-213.

Koštálová, Hana / Miková, Šárka / Stang, Jiřina (2008): Školní hodnocení žáků a studentů. Praha, Portál.

Kresic, Marijana (2006): Sprache, Sprechen und Identität. Studien zur sprachlich-medialen Konstruktion des Selbst. München, Iudicium.

Legutke, Michael K. (2013): Identität und Selbst im fremdsprachlichen Klassenzimmer. In: Burwitz-Melzer, Eva / Königs, Frank G. / Riemer, Claudia (Hgg.): Identität und Fremdsprachenlernen. Tübingen, Gunter Narr. S. 153-162.

Little, David / Perclová, Radka (2001): Evropské jazykové portfolio: príručka pro učitele a školitele. Praha, Ministerstvo školství, mládeže a tělovýchovy České republiky.

Moschner, Barbara / Dickhäuser, Oliver (2010): Selbstkonzept. In: Rost, Detlef H. (Hg.): Handwörterbuch Pädagogische Psychologie. Weinheim und Basel, Beltz Verlag. S. 762-767.

Neisser, Ulrich (1988): Five kinds of self-knowledge. In: Philosophical Psychology 1. S. 35-59.

Neisser, Ulrich / Jopling, David. A (Hgg.) (1997): The conceptual Self in Context: Culture, Experience, Self-understanding. Cambridge, Cambridge University Press.

Oppenrieder, Wilhelm / Thurmair, Maria (2003): Sprachidentität im Kontext von Mehrsprachigkeit. In: Anich, Nina / Thim-Mabrey, Christiane (Hgg.): Sprachidentität Identität durch Sprache. Tübingen, Gunter Narr. S. 39-60.

Panadero, Ernesto / Alonso-Tapia, Jesús (2013): Self-assessment: Theoretical and Practical Connotations. When it happens, how is it acquired and what to do to develop it in our students. In: Electronic Journal of Research in Educational Psychology 11/2/2013. S. 551-576.

Riemer, Claudia (2013): Konzepte und Selbstkonzepte von Fremdsprachenlernenden. In: Burwitz-Melzer, Eva / Königs, Frank G. / Riemer, Claudia (Hgg.): Identität und Fremdsprachenlernen. Tübingen, Gunter Narr. S. 223-232.

Roche, Jörg (2013): Identität und Sprache. In: Burwitz-Melzer, Eva / Königs, Frank G. / Riemer, Claudia (Hgg.): Identität und Fremdsprachenlernen. Tübingen, Gunter Narr. S. 233-246.

Slavík, Jan (1999): Hodnocení v současné škole. Praha, Portál.

Stranovská, Eva (2012): Teoretické a pragmatické základy psycholingvistiky. Brno, MSD.

Tassinari, Maria Giovanna (2010): Autonomes Fremdsprachenlernen: Komponenten, Kompetenzen, Strategien. Frankfurt am Main, Peter Lang.

Vollmer, Helmut J. (2007): Leistungsmessung, Leistungskontrolle, Selbstbeurteilung: Überblick. In: Bausch, Karl-Richard / Christ, Herbert / Krumm, Hans-Jürgen (Hgg.): Handbuch Fremdsprachenunterricht. Tübingen, A. Francke. S. 365-370. 
Weskamp, Ralf (2007): Self-assessment/Selbstkontrolle, Selbsteinschätzung und -einstufung. In: Bausch, Karl-Richard / Christ, Herbert / Krumm, Hans-Jürgen (Hgg.): Handbuch Fremdsprachenunterricht. Tübingen, A. Francke. S. 382-384.

Wilkening, Monika (2013): Selbst- und Partnerevaluation unter Schülern. Weinheim und Basel, Beltz Verlag.

Wolff, Dieter (1994): Der Konstruktivismus: Ein neues Paradigma in der Fremdsprachendidaktik? In: Die Neueren Sprachen 93. S. 407-429.

Jana Veličková

Lehrstuhl für deutsche Sprache und Literatur Masaryk-Universität Brno/Tschechien

Poříčí $7 / 9$

60300 Brno

E-Mail: velickova@ped.muni.cz 